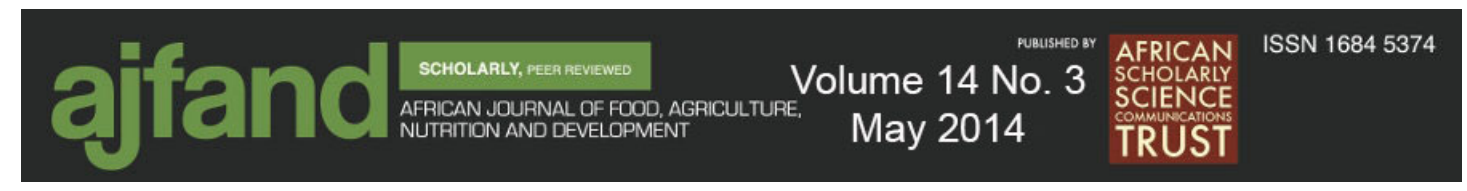

\author{
Review Article
}

\title{
DOES THE DISTRIBUTION OF READY TO USE FOOD PRODUCTS FOR THE PREVENTION OF UNDERNUTRITION MEET THE ULTIMATE NEEDS OF THE BENEFICIARY?
}

$$
\text { McLellan } \mathbf{A}^{\mathbf{1}^{*}}
$$

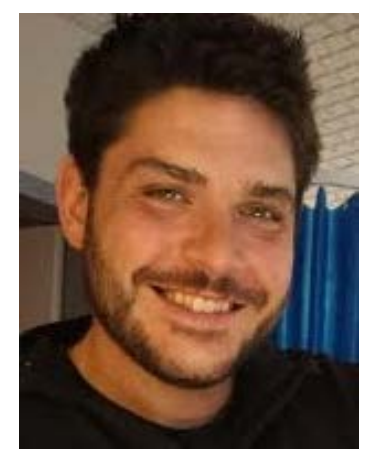

Andrew McLellan

*Corresponding author’s email: mclell00@hotmail.com

${ }^{1}$ The author is a lecturer at the University of Zimbabwe, Department of Nursing Science, and provides primary health care, HIV/AIDS treatment and care, and community management of acute malnutrition to peri-urban population in the extensions of Harare. 


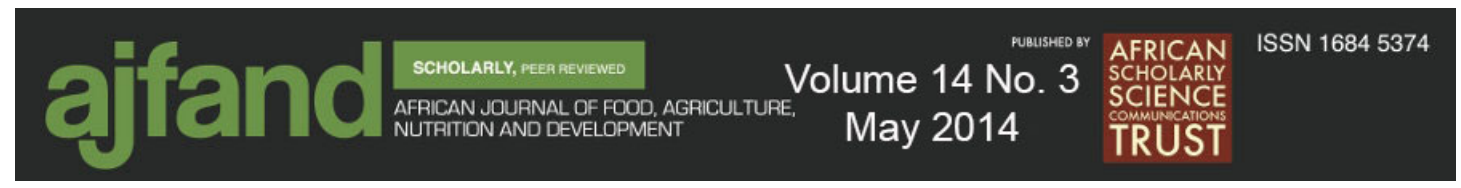

\begin{abstract}
Various nutrition products are increasingly being used to treat undernutrition in humanitarian and development interventions. The United Nations (UN), International Non-Governmental Organizations (INGOs), and donor agencies have increased their promotion of Ready to Use Foods (RUF) -both therapeutic and supplementary- for the prevention of undernutrition. Undernutrition is a major global public health problem and remains a leading cause of death of children worldwide. Irreversible changes on normal physical growth and cognitive development in undernourished children can have lasting consequences in terms of increased susceptibility to disease, threatened livelihoods, and shortened lifespans. Undernutrition is commonly found in low-income groups, in developing countries, and is strongly associated with poverty. Major consensus exists regarding the use of Ready to Use Therapeutic Foods (RUTF) in the treatment of Severe and Acute Malnutrition (SAM). There is, however, less evidence to support the use of RUF in the prevention of undernutrition. Some humanitarian actors worry that too great of a focus on the distribution of RUF in the prevention of undernutrition will detract from investments in preventative long-term and sustainable interventions that address the multiple causes of undernutrition and food insecurity. Sustainable interventions lie in the development of more productive local agricultural, a more diverse mix of nutritious crops, and a greater public awareness regarding feasible, low-cost, and local approaches to a healthy diet. RUF has little to no role to play in the prevention of undernutrition. Interventions implemented to prevent undernutrition need to focus on programs and not products as essential components of their design. This article examines RUF and its current indications for use, the evidence for the use of RUF in the prevention of undernutrition, and advocates for humanitarian actors and donor agencies to strongly support sustainable and empowering interventions over the importation and distribution of prepackaged foreign made solutions.
\end{abstract}

Key words: Undernutrition, Prevention, RUF, Humanitarian, Sustainability 


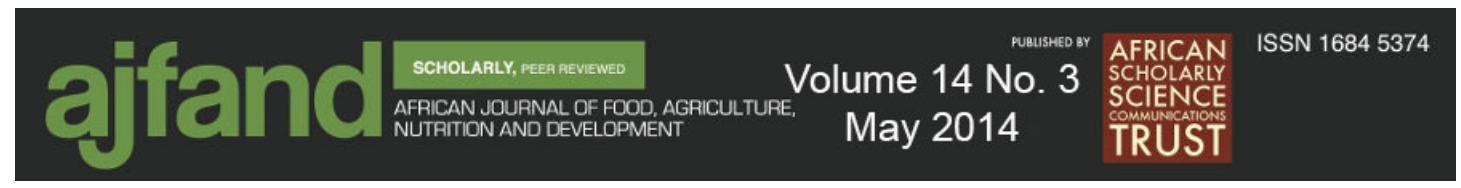

\section{INTRODUCTION}

"Give a man a fish and he eats for a day, teach him to fish and he eats for a lifetime"(Chinese proverb)

A range of nutrition products is increasingly being used to tackle undernutrition in the humanitarian and development communities. Over the last 10 years there has been a significant increase in the number of products available on the market [1]. Currently the United Nations (UN), International Non-Governmental Organizations (INGOs), and donor agencies have increased their promotion of Ready to Use Foods (RUF) -both therapeutic and supplementary- in resource poor settings (mainly in Africa and parts of Asia) for the prevention of undernutrition [1]. This article will examine RUF and its current indications for use, the evidence for RUF use in the prevention of undernutrition, and the reasons for humanitarian actors and donor agencies to refocus their attention on sustainable and empowering interventions over the importation of prepackaged foreign- made solutions. Through this exploration it will be argued that the distribution of RUF products (other than for the treatment of Severe Acute Malnutrition -SAM) can disempower communities and create unsustainable dependencies.

\section{BACKGROUND ON UNDERNUTRITION}

Undernutrition is a major global public health problem [2]. Undernutrition is caused primarily by inadequate intake of dietary energy, disease, or both. The undernutrition in children can cause irreversible changes on normal physical growth and cognitive development which may contribute to shortened lifespans, increased susceptibility to disease and threatened livelihoods [2]. Undernutrition remains a leading cause of death of young children throughout the world and is commonly found in low-income groups, in developing countries, and it is strongly associated with poverty [3]. Undernutrition includes the concepts of acute malnutrition, chronic malnutrition, and micronutrient malnutrition.

This article focuses on the undernutrition of children and specifically on the prevention of mild to Moderate Acute Malnutrition (MAM) and chronic malnutrition. Acute malnutrition can develop when an individual suffers from current severe nutritional restrictions, a recent bout of illness, inappropriate childcare practices or, more often, a combination of these factors [3]. It is characterized by weight loss, resulting in low weight for height, and/or bilateral oedema, and, in its severe form, can lead to death. Moderate acute malnutrition carries a risk of death three times greater than that of wellnourished children [3]. Chronic malnutrition (stunting) results from the same underlying causes as acute malnutrition but occurs more gradually over a longer-term. Risk of death in chronic malnutrition is two to four times greater than in well-nourished children [3]. The prevalence of chronic malnutrition is estimated to be $90 \%$ of worldwide undernutrition while acute malnutrition is estimated to have a prevalence of $10 \%[4]$. 


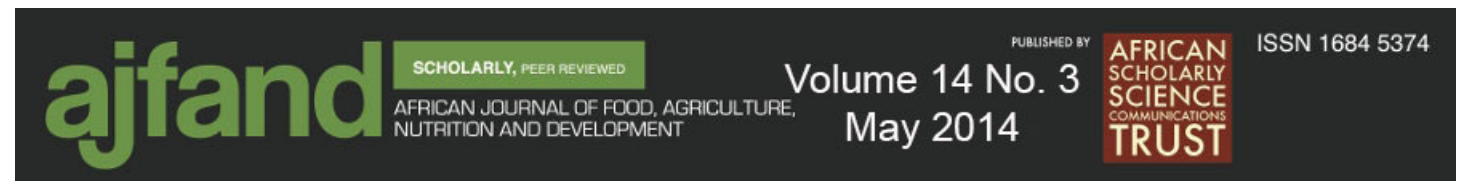

\section{READY TO USE FOOD}

The first Ready to Use Therapeutic Foods (RUTF) - brand name plumpy'nut- was developed in 1996 in France by a company called Nutriset [5]. Nutriset and its franchisees are the biggest manufacturers of RUTFs and Ready to Use Supplementary Foods (RUSF) - brand name plumpy'doz- in the world [5]. United Nation agencies are the major purchasers of RUTF and RUSF worldwide [6]. The United Nations International Children's Emergency Fund (UNICEF) is responsible for the treatment of malnourished children and provides RUTF as part of their treatment model in over 50 countries [6]. The World Food Program (WFP) is responsible for supplementary feeding for which it provides RUSFs in over 23 countries [6]. Food aid can be widely distributed to an entire population in an emergency, targeted as supplemental or therapeutic food to vulnerable groups, or provided as complementary food to improve people's usual diets [2]. Ready to use supplemental foods are developed to supplement a usual diet, with the goal to prevent or treat MAM and chronic malnutrition [6].

The use of RUTF for the treatment of SAM is largely uncontroversial [7].Ready to use therapeutic foods (RUTF) have been proven to be efficacious for the treatment of SAM without medical complications [8]. Ready to use therapeutic foods (RUTF) treatment has enabled the majority of cases of SAM to move form expensive inpatient treatment to community-based treatment programs [7]. The UN has endorsed RUF for Community- Based Management of Malnutrition (CMAM) in emergency settings; however, there is no guidance on its use in non-emergency settings, or on the role of nutrition products in the prevention of MAM or chronic malnutrition [6].

\section{EVIDENCE FOR THE USE OF RUF IN PREVENTION}

Although worldwide consensus exists regarding the treatment of SAM with RUFT, prevention is a different matter. In 2006, Medecine sans Frontirere (MSF) moved beyond treating SAM with RUFT and started distributing RUTF and RUSF for the prevention of undernutrition [7]. In one district of Niger, MSF gave 80000 children between the ages 6 and 36 months RUF in what they called blanket distribution [7]. Results from the large-scale blanket distribution of RUF in Niger suggested that the product could prevent incidence of SAM in children during the hunger gap (the period between harvests) [9]. The program evaluation found that the mean weight-for-height z-score of the intervention group that received RUF was significantly higher during the hunger gap than a control group who did not receive the product; however, no difference was found in the nutritional status of the groups throughout the rest of the year [10]. Hence, the intervention had no sustainable impact when the control versus intervention groups was compared over time. Most research on the impact of RUF has concentrated on comparing one product to another or on efficacy in terms of treating and preventing acute malnutrition over the short-term [6]. No studies have set out to examine impact over the long-term (greater than 6 months) [6]. The available evidence suggests that while RUF may prevent acute malnutrition in the short- term, there is little impact in prevention over the long- term, and there is variable impact in preventing chronic malnutrition [6]. Only one study done so far using RUF has shown a significant 
impact on growth and this was an efficacy trial [11]. Program intervention evaluations using RUF has, however, shown no impact on linear growth [12]. There have been few studies on the effectiveness of programs to prevent acute and chronic malnutrition with RUF [6]. The formulation of products and the quantities given varied widely in the different studies and due to this it is difficult to recommend an evidence-based approach for the treatment and prevention of MAM or chronic malnutrition with RUF [8]. The distribution of RUTF and RUSF, however, continues for prevention of undernutrition in the absence of strong evidence supporting the practice.

\section{SUSTAINABLE AND EMPOWERING SOLUTIONS}

There are worries amongst some humanitarian actors that too great a focus on RUF will detract from investment in preventative long- term and sustainable interventions that address the different causes of undernutrition and food security [1,6]. Proponents of the use of RUF for prevention view the products with concern, seeing RUF as a temporary 'band-aid' solution that fails to address the complex underlying causes [1]. Moreover, there is concern that RUF may undermine the promotion of breastfeeding and result in changing traditional dietary patterns [1]. The introduction of RUF into communities may create the belief that packaged foods are better for children as they improve growth. Families may start to look for and purchaseRUF products instead of consuming traditionally available foods [6]. For a family to have good nutrition, RUF does not provide a long-term answer.

Humanitarian actors that favor the distribution of RUF products for prevention take a top- down approach to resolving child undernutrition and ignore many of the root causes. The blanket distribution of RUF medicalizes the very human functions of finding/growing/ and eating nutritional foods. This leaves people believing that they must depend on foreign- made products rather than depend on themselves to prevent undernutrition in their communities. Working on empowering communities with the tools and knowledge to build food security is admittedly harder and more labor intensive than the distribution of RUF. However, doing so creates sustainable solutions that in the long- term are more successful.

An innovative and locally initiated project in rural Zimbabwe (featured in the June 2013 edition of AJFAND) had teams of farmers and health professionals hold collaborative workshops in rural communities with the goal of preventing undernutrition. The team initially conducted research about the nutritional content of locally consumed foods and into the agricultural condition required for the farming of crops, vegetables and fruits in the area. The team concluded that highly nutritious and affordable foods, that are climatically well adapted and drought resistant, can be grown and incorporated into the diets of the surrounding communities. The workshops were structured to provide 1) health education regarding locally available foods; 2) a practical component -where participants took part in the production and cooking of highly nutritious and locally available foods; and 3) agricultural instruction and practice- regarding growing, natural repellents and natural fertilizers. The intervention employed an 'upstream' approach where the root causes of undernutrition were addressed and examined and attempts at 
primary prevention were made. The approach was in contrast to many tertiary interventions that generally respond with food hand-outs in response to undernutrition.The project's approach and philosophy was grounded in empowering people with the capability of self-determination and responsibility in regards to their health and food supply rather than depending on hand-outs or medical interventions. Although the intervention design did not include a formal evaluation, the incidence of undernutrition was observationally reported to have decreased at the local hospital in the months following the community workshops.

\section{CONCLUSION}

Few dispute the power of RUF in the treatment of SAM. However, there is little evidence that RUF works equally well in the prevention of undernutrition. For decades, food aid has been distributed to help combat famine, food insecurity, and undernutrition but the number of people suffering from undernutrition has remained high [2]. Moving from responding to preventing undernutrition is commendable and necessary; however, the means of prevention is in need of a strategic rethink in order to concentrate on sustainable and empowering interventions. A refocus on programs and not products is essential for successful and lasting prevention [6]. Sustainable solutions lie in the development of more productive local agricultural, a more diverse mix of nutritious crops, and a greater public awareness regarding feasible, low-cost, and local approaches to a healthy diet [4]. Ready to use food has little to no role to play in the prevention of undernutrition. Nonetheless, some humanitarian actors promote RUF as the 'magicbullet' [4]. It is true that the distribution of RUF in the prevention of undernutrition is easier to carry out when compared with community- based empowering interventions. However, beneficiaries deserve sustainable interventions that allow progress rather than stagnation and empowerment rather than dependency.

\section{ACKNOWLEDGMENTS}

The author would like to thank the staff, lecturers, and tutors of the International Diploma of Humanitarian Assistance program offered by Fordham University, New York, for their commitment to humanitarianism and their zeal to share their knowledge and experiences with others. 


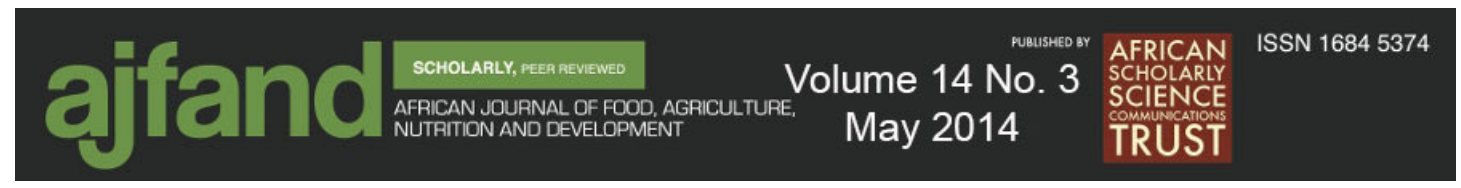

\section{REFERENCES}

1. Latham M, Jonsson U, Sterken E and G Kent RUTF Stuff. Can the Children be Saved with Fortified Peanut Paste? World Nutrition, 2011;2, 62-85.

2. Bleggi I Improving Food Aid to Improve Maternal and Child Health. Bread of the World 2012; Obtained at www.bread.org

3. Black R, Allen L, Bhutta Z, Caulfield L, deOnis M, Ezzati, M, Mathers C and J Rivera Maternal and Child Undernutrition: Global and Regional Exposures and Health Consequences. Lancet2008;371, 243-260.

4. Sachs J, Fanzo J and S Sachs Saying 'Nuts' to Hunger. Huffington Post2010; Obtained at: http://www.huffingtonpost.com/jeffrey-sachs/saying-nuts-tohunger_b_706798.html Accessed $23^{\text {rd }}$ November 2012

5. Rice A The Peanut Solution. The New York Times2010; Obtained at http://www.nytimes.com/2010/09/05/magazine/05Plumpyt.html?pagewanted=all Accessed $23^{\text {rd }}$ November 2012

6. DFID. The Use of Nutrition Products for the Prevention and Treatment of Under Nutrition. 2011; Obtained at http://hdrc.dfid.gov.uk/2011/02/the-use-ofnutrition-products-for-the-prevention-and-treatment-of-undernutrition/ Accessed $23^{\text {rd }}$ November 2012

7. $\quad$ Enserick M The Peanut Butter Debate. Science 2008;322, 36-38.

8. WHO/UNICEF/WFP/SCN Community based management of severe acute malnutrition. Joint Statement May 2007.

9. Defourny I, Minetti A, Harczi G, Doyon S, Shepherd S, Tectonidis M, Bradol J and M Golden A Large-Scale Distribution of Milk-Based Fortified Spreads: Evidence For A New Approach in Regions With High Burden of Acute Malnutrition. PLoS One 2009; 4, e5455.

10. Isanaka S, Nombela N, Djibo A, Poupard M, Van Beckhoven D, Gaboulaud V, Guerin P and RGrais Effect of Preventive Supplementation With ReadyTo-Use Therapeutic Food On The Nutritional Status, Mortality, and Morbidity of Children Aged 6 to 60 Months In Niger. Journal of the American Medical Association2009; 301, 277-285.

11. Adu-Afarwuah S, Lartey A, Brown K, Zlotkin S, Briend A and K Dewey Home Fortification of Complementary Foods With Micronutrient Supplements is Well Accepted and Has Positive Effects on Infant Iron Status in Ghana. American Journal of Clinical Nutrition2008; 87, 929-38.

12. De Pee $\mathbf{S}$ and $\mathbf{M}$ Bloem Current and Potential Role of Specially Formulated Foods and Food Supplements For Preventing Malnutrition Among 6 to 23Month-Old Children and For Treating Moderate Malnutrition Among 6 to 59 Month-Old Children. Food and Nutrition Bulletin2009; 30, (supplement). 